\title{
OBSERVATION OF THE WZ SGE-TYPE DWARF NOVA AL COM
}

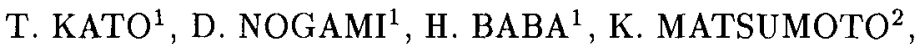 \\ J. ARIMOTO ${ }^{2}$, K. TANABE ${ }^{2}$, K. ISHIKAWA ${ }^{2}$ \\ 1. Department of Astronomy, Kyoto University, Sakyo-ku, \\ Kyoto 606-01, Japan \\ 2. Astronomical Institute, Osaka Kyoiku University, \\ Asahigaoka, Kashiwara, Osaka 582, Japan
}

\begin{abstract}
Although presently classified as a SU UMa-type dwarf nova, WZ Sge is well known as one of the most peculiar objects in that it shows only superoutbursts with exceptional duration and amplitude, and no normal outbursts. Furthermore, on its decline from the 1978 outburst, WZ Sge showed a deep temporal dip. All of these characteristics have puzzled both theoreticians and observers.

The dwarf nova AL Com was photometrically observed during the outburst in 1995 April, which occurred for the first time since 1975. The striking similarity of AL Com to WZ Sge, as demonstrated by the present observation (Fig. 1), provides plenty of material in interpreting the enigmatic nature of WZ Sge-type dwarf novae.
\end{abstract}

\section{The early stage: superhumps and 'orbital' superhumps}

In the early stage of the outburst, two distinct types of periodic variation were observed: (i) a $0.05666 \mathrm{~d}$ variation with decaying amplitude in the earliest stage of the outburst, and (ii) $0.0572 \mathrm{~d}$ superhumps which grew in $\sim 11 \mathrm{~d}$ after the onset of the outburst. We attributed the former period to the orbital period of this cataclysmic binary, which seems to be later confirmed by a period analysis of quiescent light variation. The doublehumped profile of this variation seems to preclude the explanation of a hot spot enhanced by the mass-transfer burst from the secondary. This has been believed to be the best observational evidence of a mass-transfer burst in WZ Sge, obtained during its 1978 outburst.

77

A. Evans and J. H. Wood (eds.), Cataclysmic Variables and Related Objects, 77-78.

0 1996 Kluwer Academic Publishers. Printed in the Netherlands. 


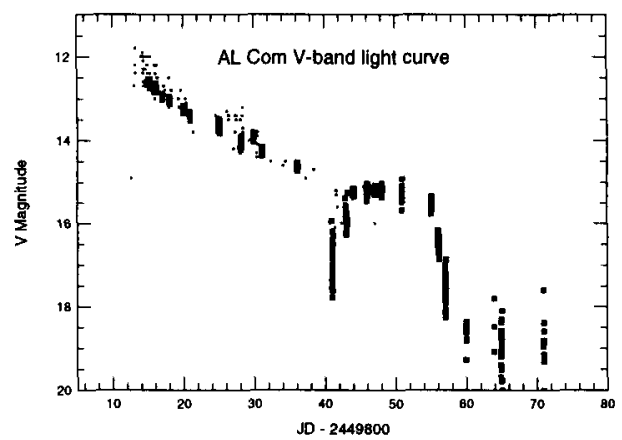

Figure 1. The overall $V$-band light curve of the 1995 outburst of AL Com. Note the exceptionally long duration of the outburst interrupted by a deep dip, both of which are clearly reminiscent of the 1978 outburst of WZ Sge.

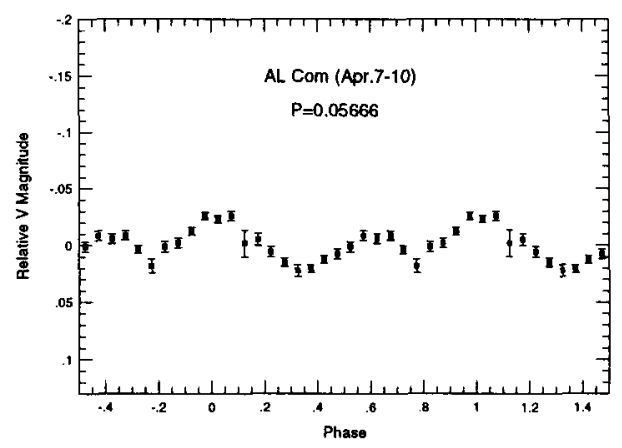

Figure 2. 'Orbital' superhumps observed in the earliest stage of the outburst. The double-humped profile of this variation seems to preclude explanation by the enhanced hot spot.

\section{After the 'dip' behavior}

The present outburst of AL Com showed a 'dip' very similar to the one observed in the 1978 outburst of WZ Sge. Non-existence of orbital humps during the recovery from the dip strongly supports the interpretation that the re-brightening was not powered by a mass-transfer event, but by the thermal instability of the accretion disk. After the dip, AL Com showed a short-lived maximum followed by a rapid decline. After this 'secondary dip' the star entered a new stage; the 'plateau phase'. The observation has shown that the superhumps again grew during this phase. We can therefore identify this stage as the start of a new superoutburst. The shortlived maximum after the main dip may represent a normal outburst which triggered this superoutburst. The peculiar outburst light curve common to WZ Sge and AL Com now seems to be naturally understood by the occurrence of two successive superoutbursts, which are powered by the usual combination of thermal and tidal disk instabilities. 